Full title: Cultivation of mycelium of the edible fungus Pleurotus ostreatus (oyster mushroom) with the use of wet sparging grain, the waste of the brewing industry.

\title{
Names of Authors
}

Georgy V. Pestsov

Anastasia V. Glazunova

Yuriy M. Atroshchenko

Roman A. Sidorov

\section{Author Affiliations}

Georgy V. Pestsov; Anastasya V. Glazunova; Yuriy M. Atroshchenko; Roman A. Sidorov L.N. Tolstoy State Pedagogical University, Tula, Russia

Georgy V. Pestsov -

All-Russian Research Institute of Phytopathology, Moscow, Russia

\section{Contact information for Corresponding Author}

Prof. Georgy V. Pestsov, apt. 3, 66A Lenin Ave., 300012 Tula, Russian Federation.

\section{georgypestsov@gmail.com}

ABSTRACT: Wet sparging grain, a waste product of brewery, was shown to be successfully utilized as a supplement to the nutrient media and substrates for both isolation of the vegetative mycelium of the Pleurotus ostreatus mushroom, as well as for its further recovery and growing the carposomes. We studied the influence of the wet sparging grain on mycelium growth. Cultivation results demonstrated that wet sparging grain is an efficient additional component to the minimal agarized nutrient media for $P$. ostreatus growth and isolation and to its common fruit body cultivation substrates. Even the depleted agaric media and substrates with addition of the sparging grain allowed intensive growth of the $P$. ostreatus mycelium and its fructifying. 
Practical Application: Addition of wet sparging grain to poor cheap substrates increases their quality for rearing the edible oyster mushroom mycelium to the level of reach substrates, and allows utilization of agricultural wastes. The technique is ready for application and patented in the Russian Federation. 


\section{Introduction}

The sparging grain is a by-product of brewing industry. It is estimated that the annual production of sparging grain accounts for about $30 \mathrm{~m} / \mathrm{n}$ tone globally. Therefore a huge volume of biomass results in a burning problem of storing and recovery of the brewing industry waste. Biological destruction of waste products produces organic compounds, which are decomposed and discharge stinking methane, spirits, carbon oxide, ammonia, indole, hydrogen sulfide and are dangerous for people. It results in changing the chemical composition of soil, moreover, toxic compounds that are the product of protein decomposition, pass through the ground and sewage, changing the $\mathrm{pH}$ of soil that is harmful for useful microorganisms. Such soil is not suitable for agriculture for a long period. All these factors cumulatively bring a harmful effect to the environment. Sparging grain looks like a thick friable mass of brown or yellowish colour. It has a sharp, caustic and special smell. Sparging grain comprises cereals particles and shell. There are several alternative variants of how to use sparging grain. They include forage and fertilizer, according to Mussatto and Roberto (2006). However, due to a high water content the grain's storage period is limited. There are some methods that allow to process the grain and to use it like a food for people because it has a high nutritive value and its availability in large volumes during the year. In farming it is used as additive to lactigenic and protein forage instead of flesh-and-bone meal for agricultural animals and poultry. Sparging grain features a high assimilability of its protein substances, lipids, anazotic extractive substances, cellulose, as Sviridov (2005) and Mussatto et al. (2006) state. 
Chemical composition of the grain depends on the raw materials, mainly breed of barley, harvest year, maltage and malt mashing conditions as well as brand and quality of other cereals used in the brewing process, according to Wilhelmson et al. (2009). Sparging grain is comprised of cellulose, (16-25\%), hemicellulose (21-35\%), lignin (11-28\%) and proteins (15-25\%), as well as amino acids connected with proteins, irreplaceable included. Also it includes a lot of vitamins, mainly biotin, choline, folic acid, folic acid, niacin, pantothenic acid, riboflavin, thiamine, pyridoxine and various minerals including calcium, magnesium, silicon, phosphorus, copper, ferrous and manganese ions, potassium, sodium, sulfur.

Different percentage of components depends on various brands of grain, harvest year, brewing conditions plus the quality and type of additives in the brewing process (Rugolo, Lechner, Mansilla, Mata, \& Rajchenberg, 2020; Mussatto et al., 2006). Sparging grain phenolic extracts featured anti-oxidative potential against DNA destruction. Sparging grain is extremely profitable as an additive to animals forage resulting in increase of milk yield, butter lipid, irreplaceable acids.

The use of the Grain as an additive to people food such as biscuits, snacks resulted in increase of their protein and cellulose (McCarthy, O'Callaghan, Piggott, FitzGerald, \& O'Brien, 2013). Sparging grain comprises a large volume of carbohydrates, protein, lipids, ash elements and therefore it can be used in various branches of agriculture. It is a valuable biologic foodstuff for mushrooms as it includes mainly lignin, cellulose, hemicellulose. It presents a source of nutrients, exactly carbohydrates, amino acids and mineral substances. There has been a build-up of attempts to use the Grain in the biotechnological processes of the mushrooms growing (Mussatto, 2009). High content of protein, cellulose, hemicellulose and lignin, which are main components of mushrooms vital functions, presents a special 
interest. Waste products comprising cellulose can be actually used by xylophages, which synthesize enzymes of peroxides and Cu-dependent laccase. Pleurotus ostreatus is capable to break lignin to its complete mineralization due to laccase secretion, as well as $\mathrm{Mn}$ dependent aryl-alcohol oxydases (Wang, Sakoda, \& Suzuki, 2001). Nitrous compounds are a basis for proteins that is main part of a protoplasm. They play an important role in metabolism of mushrooms. Nitrate and ammonium salts are the main source of inorganic nitrogen salts for mushrooms. The nutritious value of various amino acids differs and depends on both the content and molecule configuration. The use of amino acids is a special feature of a mushroom strain. It is due to a great variability of a gene pool of the species $P$. ostreatus (Bysko and Dudka, 1987; Salihu \& Muntari, 2011).

Through a large number of nutrients, macro- and micro- elements, the grain can be considered as a potential component suitable for generating special mediums and substrates intended for growing mycelium and carposomes of edible mushrooms. Recently the development of cultivating the wood-attacking fungus is in an appreciable progress globally in many climatic zones. Currently they cultivate only ten Pleurotus species out of twenty ones. Pleurotus ostreatus get cultivated industrially in Russia is the most known among them. The $P$. ostreatus species is popular and wildly cultivated worldwide. This species became popular due to its relatively easy and affordable technology of production. The Pleurotus species are effective destroyers of lignin and can grow on agricultural waste of different type. The $P$. ostreatus carposomes are the source of protein, minerals, (Ca, P, Fe, $\mathrm{K}$ and $\mathrm{Na}$ ), vitamin $\mathrm{C}$, thiamine, riboflavin, folic acid, niacin, potassium, making them an ideal food for hypertensive and heart patients (Patil, Ahmed, Telang, \& Baig, 2010). 
To avoid main problem of the mushrooms production, one should provide optimal conditions for cultivation of mycelium and stable harvest of carpospores.

It is important to provide a high growth rate of the vegetative mycelium as well as its resistance to a competitive micro flora, particularly at the initial stage of growth. The yield of the $P$. ostreatus crop depends on the substrate degradation and its content of nutrients plus the ratio of its major nourishing mineral elements of carbon, nitrogen, and phosphorus. Micro-elements play an important role, and their shortage in the mushrooms cells can lead to a deceleration of the oxidation-reduction processes that are necessary for normal growth and development. It should be remind that cations of magnesium and copper are of high importance in the process of breathing. Therefore, the use of inorganic and organic additives is highly potential in the growth of the mushrooms mycelium. The effect of various additives is well analyzed on the plants. And it is known that they can increase the harvest and act as an anti-stress factor under adverse environmental conditions (Minasbekyan, Nerkararyan, Nanagulyan, \& Avagyan, 2010). The literature data shows that the sparging grain is successfully used as an additive to the substrate for the growth of the Pleurotus ostreatus carposomes. The results of the mushrooms cultivation demonstrated that when the sparging grain was used along, there were few carposomes. The biologic effectiveness was increased greatly in case of wheat bran (45\%). The chemical analysis of the $P$. ostreatus carposomes revealed that the carposomes' harvest on the substrate of the sparging grain featured crucially higher nourishing value than the carposomes grown on the other types of the substrates. The total content of amino acids was also higher (Mussatto, 2009). The present mushroom cultivation suffers the urgent problem of how to get the competitive mycelium of a good quality that can be solved through the use of additives. The sparging grain addition at the initial stage (into the Petri dish with agar medium) will help the 
mycelium culture further to overcome stresses after the passage on a solid substrate containing cellulose. All this will result in a high harvest, and the waste of brewing and agriculture will be used for cultivation of edible mushrooms.

\section{Materials and Methods}

There researches were carried out concerning selection of nutrient medium and substrates with addition of brewing waste for cultivation of edible mushrooms. The experiment was performed with the use of an autoclave, a microbiological box, standard tools and dishes used in the process of microbiological studies. Pleurotus ostreatus (Jacq.: Fr.) Kumm. basidiomycete was chosen as a research object. We studied the way how to obtain the sowing mycelium of $P$. ostreatus with the use of the sparging grain. Initially pure mushroom culture was get by sowing of the mushroom pieces with sterilized surfaces of $1 \mathrm{~cm}^{3}$ size on a solid sterilized nutrient medium with agar in a Petri dish.

To select mycelium, we used five types of nutrient medium. The nutrient medium number one presents a medium based on wheat and prepared as follows: $1000 \mathrm{ml}$ pour into $250 \mathrm{~g}$ of cereals and boil it for $30 \mathrm{~min}$, thereafter the liquid is subjected to filtration. Then pour $20 \mathrm{~g}$ of agar and $10 \mathrm{~g}$ of sucrose, heat mixture till complete dissolving.

The medium number two is a wheat medium without sugar with agar: $1000 \mathrm{ml}$ of water is poured into $200 \mathrm{~g}$ of washed and sliced potatoes, pored and boiled during $40 \mathrm{~min}$. Thereafter it is subjected to filtration. Some additional water was added into the filtrate up to $1000 \mathrm{ml}$, and $20 \mathrm{~g}$ of agar with $20 \mathrm{~g}$ of sucrose, heated till complete dissolving. The experimental nutrient medium number three, with the use of grain, is comprised of $20 \mathrm{~g}$ of agar-agar, 100g of fresh grain per $1 \mathrm{~L}$ of water (Glazunova, Pestsov \& Sidorov, 2020). 
The Czapek-Dox medium (number five) consists of $30 \mathrm{~g}$ sucrose, $2 \mathrm{~g}$ sodium nitrate, $1 \mathrm{~g}$ sodium monohydrate phosphate, $0.5 \mathrm{~g}$ magnesium sulphate, $0.5 \mathrm{~g}$ potassium chloride, 0.01 $\mathrm{g}$ ferric sulphate, $15 \mathrm{~g}$ agar, and $1000 \mathrm{ml}$ distilled water.

The nutrient media were sterilized in the autoclave at 1.2 atmosphere during 45 minutes. The cultivation was carried out in the thermostat at $+24{ }^{\circ} \mathrm{C}$. The mycelium appeared at the surface of the carposome by a 2 nd or 3rd day. The dynamic rate of growth of mycelium was determined.

When the vegetative mycelium of the mushroom appeared, it was transferred under sterility conditions to different containers onto sterile nutrient media. To provide mass reproduction, used nutrient media with different content were used, six types in total. The potato-sucrose agar comprising $20 \mathrm{~g}$ - sucrose, $20 \mathrm{~g}$ - agar, $1 \mathrm{I}$-distilled water, was used as a reference. As a control, depleted agar was used $(20 \mathrm{~g}$ of agar was dissolved in $100 \mathrm{ml}$ of distilled water). The potato and the minimal agar with addition of the grain ( $20 \mathrm{~g} \mathrm{per} 1000$ $\mathrm{ml}$ ) and the Czapek-Dox medium were used in experimental series. The nutrient media sterilization was performed in the autoclave at $1.2 \mathrm{~atm}$ during $45 \mathrm{~min}$. Mycelium cultivation was performed in an incubator at $24^{\circ} \mathrm{C}$.

Thereafter, mycelium was sub-cultured under sterile conditions onto substrates of different content. The grain-based substrate we used as a standard. $100 \mathrm{~g}$ of cereals were filled with $250 \mathrm{ml}$ of water and boiled for 15-20 min on a slack fire. After cooking, the remained water was poured off, and $1 \mathrm{~g}$ of gypsum and $0.3 \mathrm{~g}$ of chalk were added.

For the purpose of control, a substrate based on a straw was used. It was prepared in the following way: chopped straw was steamed in water at $65-70{ }^{\circ} \mathrm{C}$ during $40 \mathrm{~min}$. There were used experimental substrates based on a straw, seeds and buckwheat husks with 
addition of the grain in the ratio $1: 1$. The nutrient media sterilization was performed in the autoclave at $1.2 \mathrm{~atm}$ during $60 \mathrm{~min}$.

After cooling the substrate was inoculated with mycelium and placed into an incubator at the temperature of $+24^{\circ} \mathrm{C}$ for 7 days. The measurements of a colony diameter were performed on the $3 \mathrm{rd}, 5$ th, and 7 th day after sawing. The results of the experiments were analyzed and statistically processed using JASP v.0.14.1 software.

\section{Results and Discussion}

At the first stage we selected the nutrient media for extracting mycelium of the oyster mushroom. For its isolation, we took parts of the carposome and put them into six types of nutrient media (Fig. 1).

In result we found that the most optimal media for isolation of the $P$. ostreatus mycelium are the media based on the wet brewers' draff, with addition of sucrose, or without it. On the seventh day, the mycelium diameter was $27 \%$ (wet brewers' draff plus sucrose) or $25 \%$ (wet brewers' draff only) larger than on the standard medium, the potato-sucrose agar. Insignificance of the difference in the growth rate brings us to the conclusion that the variant of $P$. ostreatus mycelium cultivation on wet brewers' draff without sucrose for its subsequent isolation is the most perspective considering its economic efficiency.

On the next stage we carried out the selection of the nutrient media for cultivation of the vegetating mycelium of the oyster mushroom. For that, we placed sterile mycelium on nutrient media of different composition (Fig. 2)

In result, the most optimal nutrient medium for vegetative mycelium was the minimal agar with organic supplements, including wet brewers' draff. On it, on the final, seventh, day, the mycelium occupied $9 \%$ more square of the Petri dish than on potato agar. At the early growth stages, mycelium demonstrates good growth on potato agar: on the day 5 the 
diameter of the colony reached $65 \mathrm{~mm}$. However, by the day 7, its growth speed considerably decreases and concedes the minimal agar with brewers' draff. Thus, this latter one is the best for cultivation of vegetating mycelium. On the nutrient medium of this type it forms creeping mycelium.

For obtaining the stock mycelium culture, the pure culture was reinoculated onto nutrient substrates of a different composition in vials or Petri dishes, for studying the mycelium growth speed (Fig. 3).

The experiment revealed that the optimal nutrient substrates for growing of the stock mycelium culture are those based on the wet brewer's draff with addition of cellulose containing residues. The grain-based substrate was used as a standard, according to existing techniques for rearing the stock culture (Gregori, Svagelj, Pahor, Berovic, \& Pohleven, 2008) The nutrient substrate based on the wet brewers' draff, provides the highest radial mycelium growth speed. The energetic properties of the straw substrate are low compared to it, so it provides low mycelium growth speed.

\section{Conclusions}

The use of the wet brewers' draff as a supplement to the nutrient media and substrates for mycelium growing and isolation will allow reducing the period of its adaptation to the novel growing conditions. The use of the wet grain brewers' draff allows isolation of the quality mycelium out of the $P$. ostreatus carposomes and increase accumulation of its biomass. Also, the use of this brewery waste product as a main substrate for obtaining the mycelial biomass will considerably decrease the production costs.

\section{Financing}


The present study was carried out in the framework of the grant by the Tula Region government in the field of science and technology in the year 2020, Development of the method for the brewery waste utilization using the oyster mushroom fungus, according to the contract \# ДС/165 of $10 / 29 / 2020$.

\section{Conflict of Interests}

Authors declare no conflict of interests.

\section{References}

Bysko N.A., Dudka I.A., (1987). [Biology and cultivation of edible mushrooms of the genus Pleurotus] - In Russian. Naukova Dumka, Kiev.

Glazunova, A.V., Pestsov, V.G., Sidorov, R.A. (2020). [Cultivation of the seeding mycelium of the mushroom Pleurotus ostreatus (oyster mushroom) with the use of wet brewer's draff, the waste product of the brewing industry.] - In Russian. Russian Federation Patent \# RU2732832C1 effective on 23.09.2020. Patent Bull., 27.

Gregori, A., Svagelj, M., Pahor, B., Berovic, M., \& Pohleven, F. (2008). The use of spent brewery grains for Pleurotus ostreatus cultivation and enzyme production. New Biotechnology, 25, 2-3. https://doi.org/10.1016/i.nbt.2008.08.003

McCarthy, A.L., O'Callaghan, Y., C. Piggott, C., FitzGerald, R., \& O'Brien, N. (2013). Brewers' spent grain; bioactivity of phenolic component, its role in animal nutrition and potential for incorporation in functional foods: a review. Proceedings of The Nutrition Society, 72(1), 117125. http://dx.doi.org/10.1017/S0029665112002820

Minasbekyan, L. A., Nerkararyan, A. V., Nanagulyan, S. G., \& Avagyan I. A. (2010). Changes of peroxidase activity in the culture of Pleurotus ostreatus mushroomunder influence of $\mathrm{mm}$ waves. Immunopathology, allergology, infectology, 2, 47-54.

Mussatto, S. (2009). Biotechnological Potential of Brewing Industry By-Products (pp. 313326). Biotechnology for Agro-Industrial Residues Utilization, Springer.

http://dx.doi.org/10.1007/978-1-4020-9942-7_16

Mussatto, S., Roberto, I. (2006). Chemical characterization and liberation of pentose sugars from brewer's spent grain. Journal of Chemical Technology \& Biotechnology, 81, 268-274. http://dx.doi.org/10.1002/jctb.1374

Mussatto, S., Roberto, I., \& Dragone, G. (2006). Brewers' Spent Grain: Generation, Characteristics and Potential Applications. Journal of Cereal Science, 43(1), 1-14. 
Patil, Shyam Sopanrao, Ahmed, Syed Abrar, Telang, Suresh Manoharrao, Baig, Vaseem Mushtaq Mirza. (2010). The nutritional value of Pleurotus ostreatus (Jacq.:Fr.) Kumm. cultivated on different lignocellulosic agrowastes. Innovative Romanian Food Biotechnology, 7, 66-76.

Rugolo, M., Lechner, B., Mansilla, R., Mata, G., Rajchenberg, M. (2020). Evaluation of Pleurotus ostreatus basidiomes production on Pinus sawdust and other agricultural and forestry wastes from Patagonia. Maderas. Ciencia y Tecnologia, 22(4), 517-526. https://doi.org/10.4067/s0718-221x2020005000410

Salihu A., Muntari B. (2011). Brewer's spent grain: A review of its potentials and applications. African journal of biotechnology, 10(17), 324-331.

Sviridov, D.A., Gernet, M.V, Kobelev, K.V. (2005). [Brewer's draff in the production of protein concentrates].- In Russian, Beer and Drinks. 6, 28-29. - In Russian.

Wang D., Sakoda A., Suzuki, M. (2001). Biological efficiency and nutritional value of Pleurotus ostreatus cultivated on spent beer grain. Bioresour. Technol., 78(3), 293-300. https://doi.org/10.1016/S0960-8524(01)00002-5

Wilhelmson, A., Lehtinen, P., Weymarn, N., Itävaara, M. \& Sibakov, J. (2009) Future applications for brewers' spent grain. New Food Magazine, 3. 


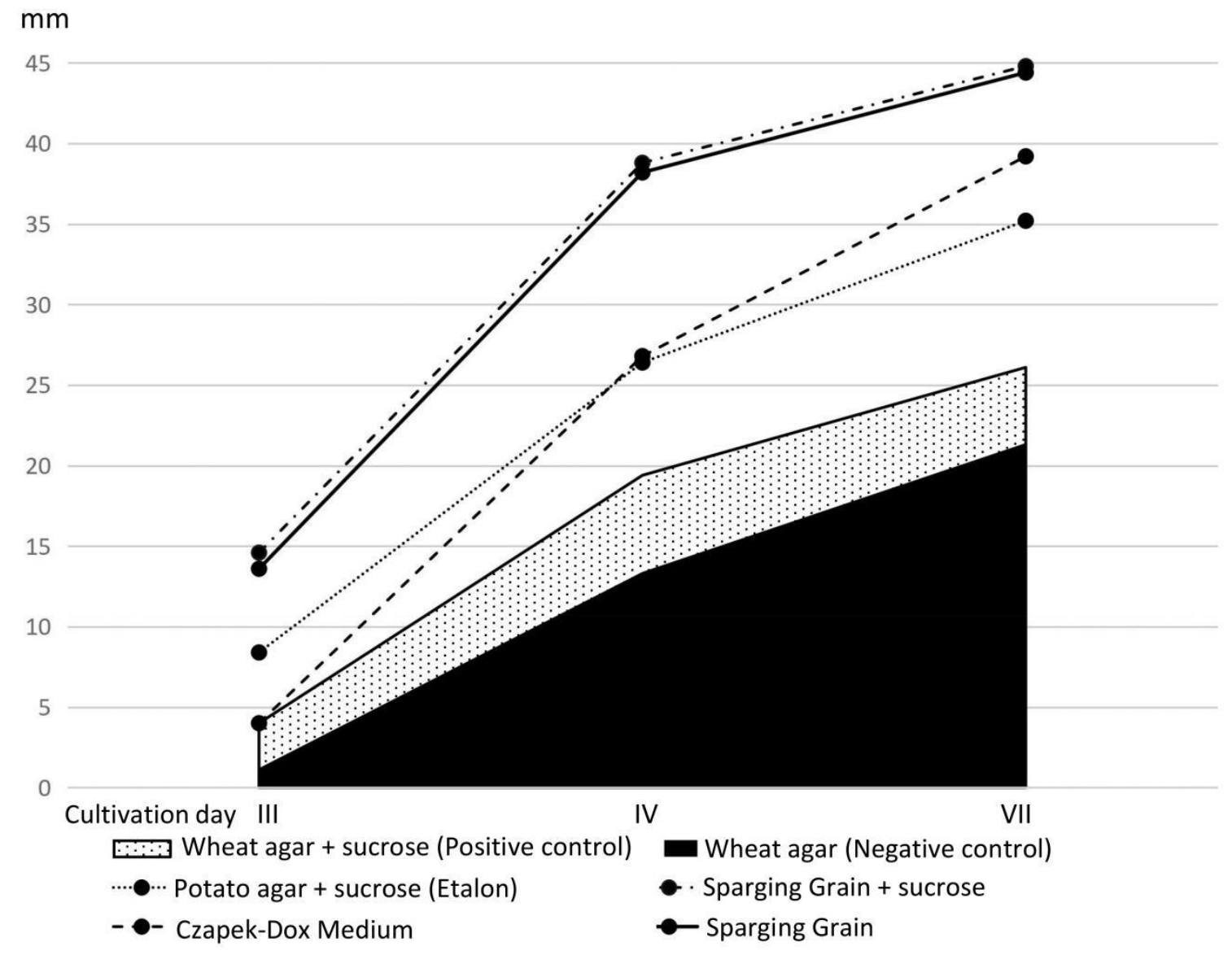

Figure 1. Radial mycelium growth on agarized media with sucrose. 


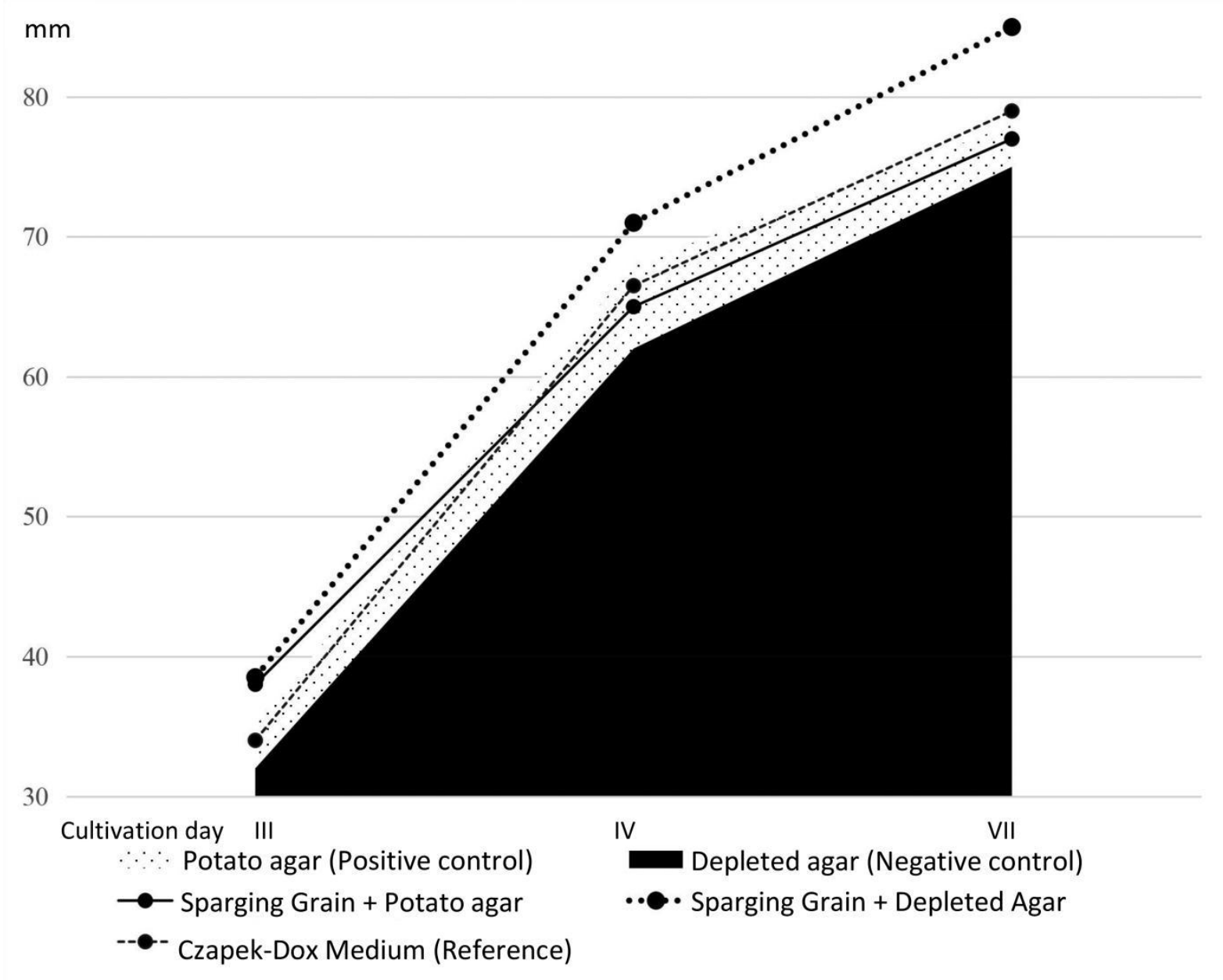

Figure 2. Radial mycelium growth on agarized nutrient media. 


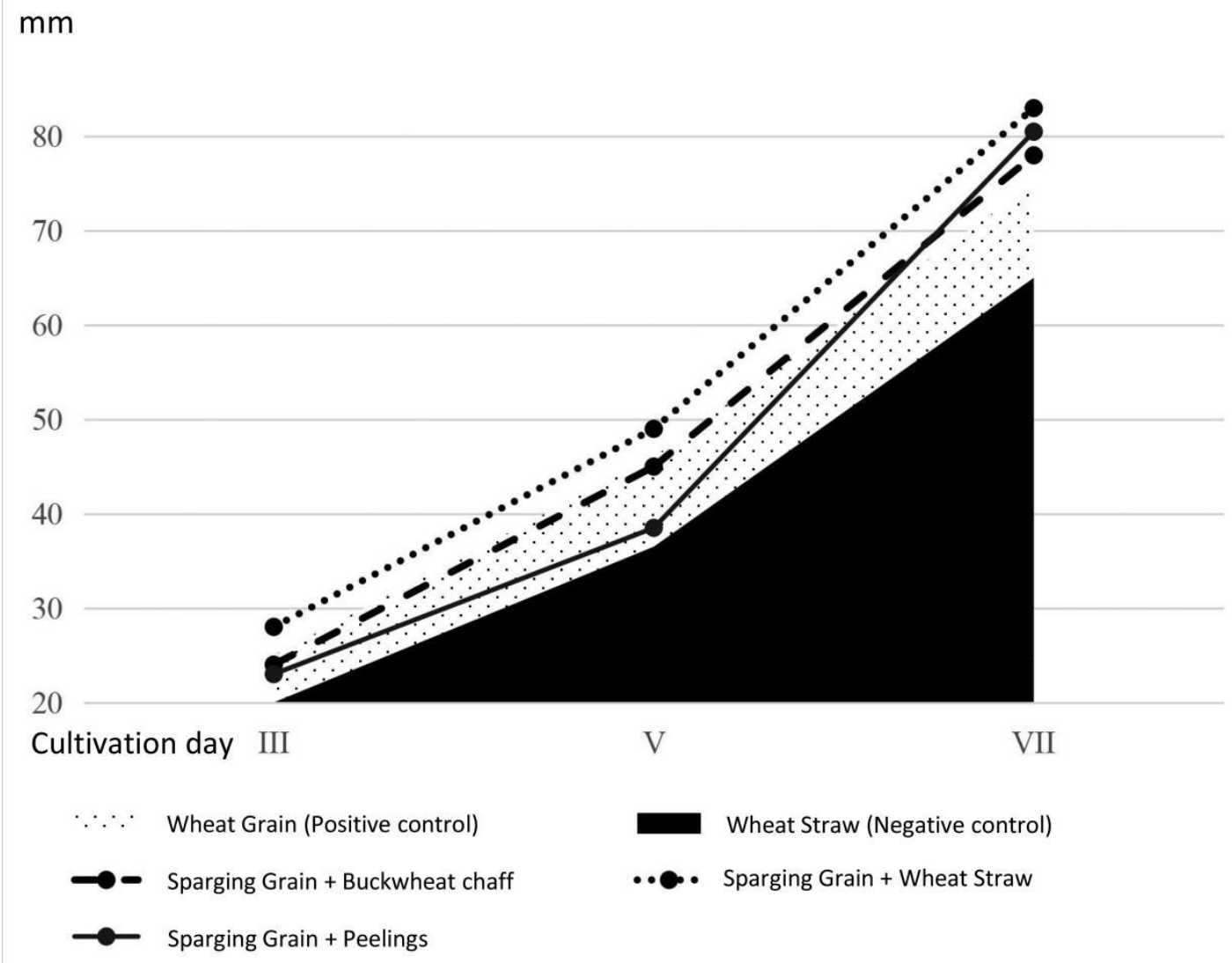

Figure 3. Radial mycelium growth on agricultural waist mass cultivation substrates. 